\title{
Analysis and Synthesis of Latin Dance Using Motion Capture Data
}

\author{
Noriko Nagata ${ }^{1}$, Kazutaka Okumoto ${ }^{1}$, Daisuke Iwai ${ }^{2}$, \\ Felipe Toro ${ }^{2}$, and Seiji Inokuchi ${ }^{3}$ \\ 1 School of Science and Technology, Kwansei Gakuin University, \\ 2-1 Gakuen, Sanda, Hyogo 669-1337, Japan \\ \{nagata, okumotokazutaka\}@ksc.kwansei.ac.jp \\ http://ist.ksc.kwansei.ac.jp/〜nagata/ \\ 2 Graduate School of Engineering Science, Osaka University, \\ 1-3 Machikaneyama, Toyonaka, Osaka 560-8531, Japan \\ \{iwai@sens, toro@yachi-lab\}.es.osaka-u.ac.jp \\ 3 Faculty of Human and Social Environment, Hiroshima International University, \\ 555-36 Gakuendai, Kurose-cho, Kamo-Gun, Hiroshima 724-0695 Japan \\ inokuchi@ieee.org
}

\begin{abstract}
This paper presents an analysis of natural movement in Latin dance and a synthesis of dance motions making use of the outcome. The isolation movement of shoulders and hips in Latin dance was extracted quantitatively, and a dance animation with different isolation levels was synthesized, using a motion graph editor.
\end{abstract}

\section{Introduction}

With the rapid progress in the architectural technology of virtual environments, further research and development in technology to express the natural movement of virtual humans is being called for, from the aspects of quality and cost. In particular, in educational contents, such as navigation and sports science, and in industrial applications such as design support, and in the field of interactive media, technology which can synthesize and edit movement and technology which can form databases for motion data that already exists will be necessary.

We have extracted the characteristics inherent in various human motions and are researching animation creation technology making use of this [1]2]. This paper discusses the extraction of natural movement in Latin dance and the creation of an animation making use of the outcome. In dance, it is often said that the Japanese do not have as good rhythmical sense as foreigners. In particular, as expressed by the expression "good tempo", Latin dances are full of rhythm and motion. There are various causes for this difference such as cultural background and we cannot jump to conclusions, but there is a finding [1] that a motion unfamiliar to the Japanese people, called isolation, is involved. Through our advanced two-dimensional research, we have confirmed a phase difference in the movement of shoulders and hips which is a characteristic of the movement of Latin people. Here, in order to further conduct precision analysis, we obtained Latin dance 

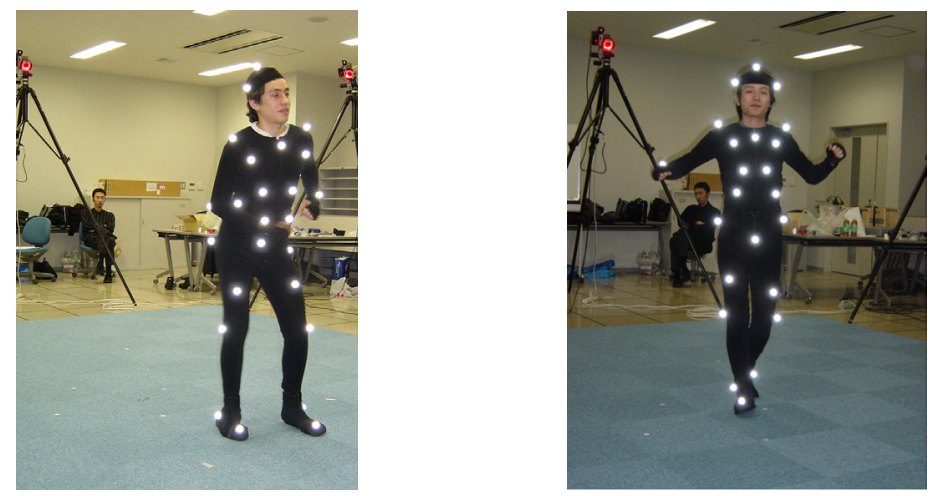

Fig. 1. Photos of subjects.

movement using motion capture and attempted to extract the difference in the characteristics of movement of people experienced in dancing (Latin people) and people inexperienced in dancing (Japanese people).

As a result, it was confirmed that isolation between the shoulder and hip takes place in a three-dimensional way in the way Latin people dance. Further, using this result, we synthesized a dance animation. To do this, we edited the bvh data of experienced people and of inexperienced people by blending them at a specified ratio using a motion graph editor [3]. In order to confirm that the created animation can express Latin movement, we made a three-dimensional display of dance animation in a human-scale virtual reality (VR) environment. The results evaluated as to whether they were like Latin movement or not.

\section{Measurement and Analysis of Dance Movement by Motion Capture}

We measured the dance motions of people experienced in Latin dance (Latins) and people inexperienced in dancing (Japanese), using motion capture. The motion capture system was composed of 8 digital cameras $(640 \times 480$ pixels, $60 \mathrm{~Hz})$ and real time capture software. The subjects were asked to wear suits with 31 marks and dance to the music. The music, a Merengue piece, which is Latin, was selected (Figure 1).

As shown in Figure 2, from the dance movement obtained, the angles of rotation of shoulders and hips (the inclinations of the line connecting the joints of both shoulders (a) and both hips (b)) were calculated. This was divided into the change in the rotational component in the plane vertical to the floor and the change in the rotational component in the horizontal plane parallel to the floor.

Figure 3 shows the angles of rotation of shoulders and hips in the vertical plane. Figure 3b shows that people inexperienced in dancing almost always assume the same angle, whereas in Figure [3a, people experienced in dancing maintain a phase difference of about 90 degrees between the shoulders and hips. 


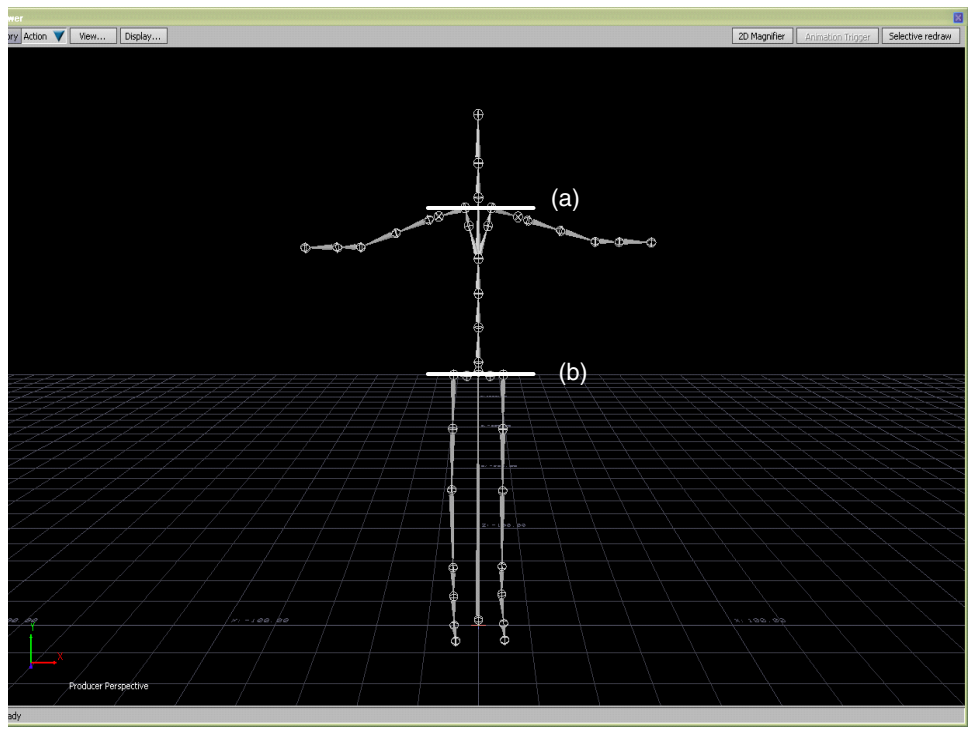

Fig. 2. The angles of rotation of shoulders and hips.
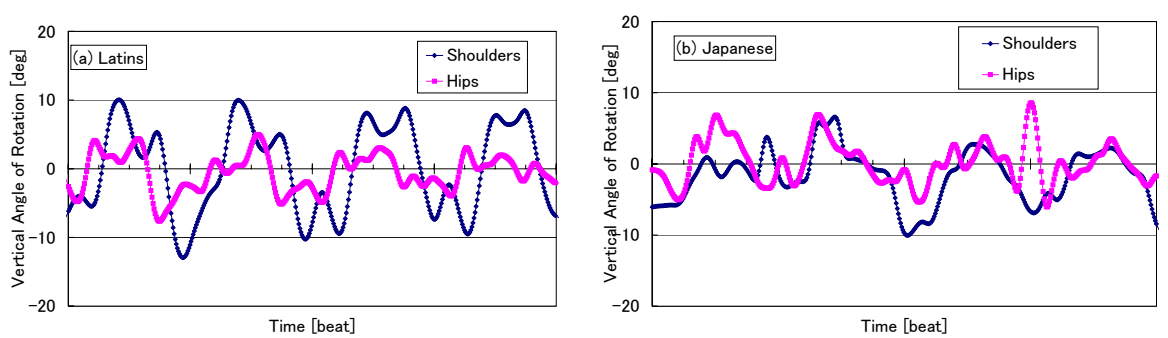

Fig. 3. The angles of rotation of shoulders and hips in the vertical plane.

Figure 4 shows the angles of rotation of shoulders and hips in the horizontal plane. Similarly Figure 4 a indicates that the movement of Latins keeps a phase difference of about 30 degrees between the shoulders and hips, no phase difference was observed in the Japanese dance.

These can be interpreted as an example of isolation between shoulders and hips moving independently, and it is considered that one of the characteristics of Latin dance has been successfully extracted.

Figure 5 shows the center of gravity of hips in the vertical plane. The up and down movements of the Latins are very small compared with the Japanese. This can also be explained as isolation, one of the characteristics of Latin dance. 

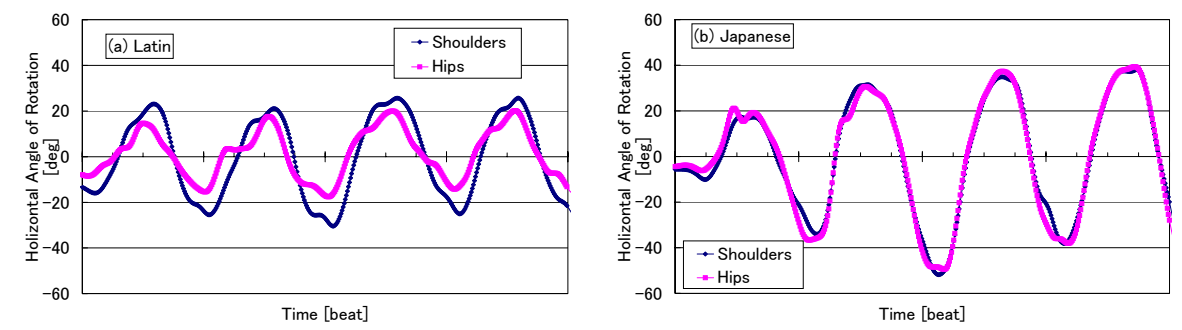

Fig. 4. The angles of rotation of shoulders and hips in the Horizontal plane.
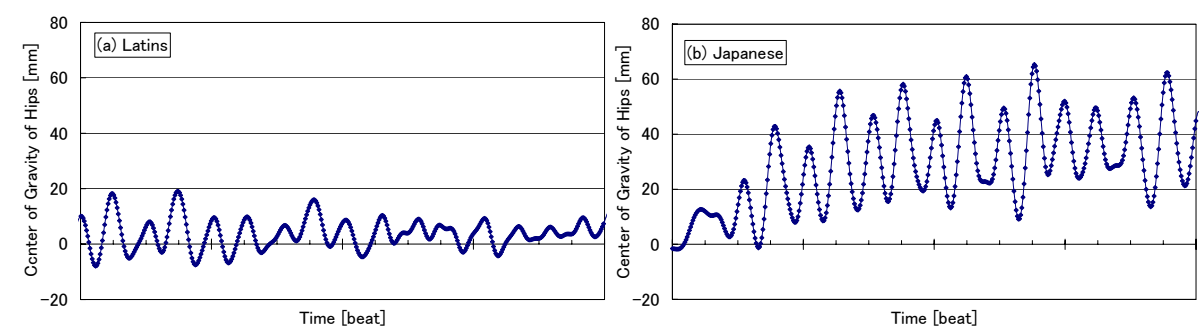

Fig. 5. The center of gravity of hips in the vertical plane.

\section{Synthesis of Dance Animation}

In order to confirm that isolation between shoulders and hips is one of the characteristics of Latin dance, we created a dance animation using our motion data and evaluated it.

We used a motion graph editor to synthesize the dance motion. The motion graph editor can connect the motion data expressed in a multiple bvh form in serial/parallel. The joint can be linearly interpolated by line blending in any ratio using the motion blend function. For instance by using a pre-measured walking movement and a running movement, it is possible to form an animation that can express the natural shift from walking to running.

Using this blending function, motion data with a difference in the isolation level between the shoulders and hips was synthesized. To be specific, the dance motions from the same cycle of the dance of an experienced person and an inexperienced person were cut out and created by blending them in a certain ratio. Then three motions, motion $\mathrm{A}$ (blend ratio 9:1), motion $\mathrm{B}$ (5:5, as shown 

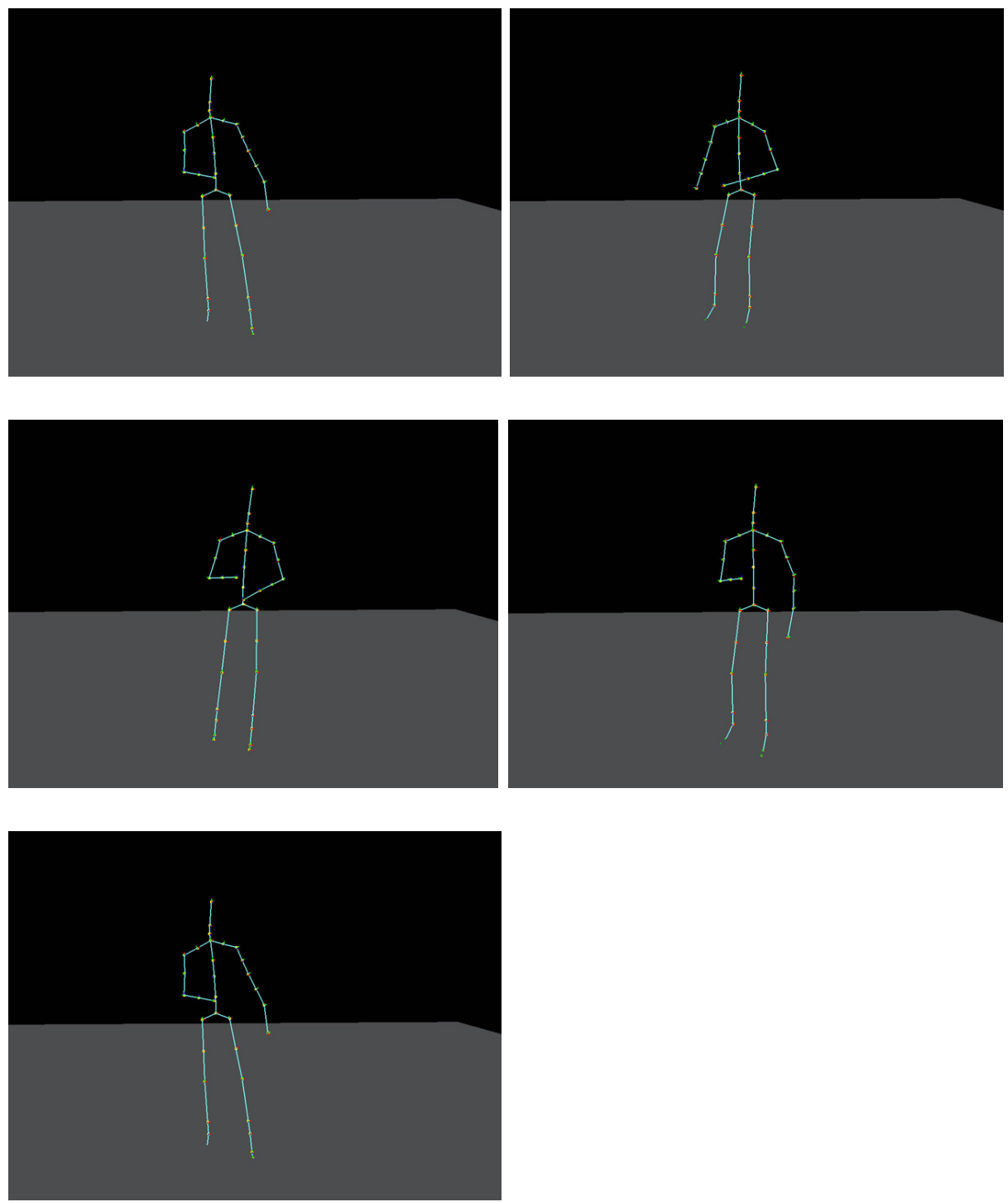

Fig. 6. An example of Synthesized Latin dance motion.

in Figure 6), motion $\mathrm{C}$ (1:9) were randomly presented to subjects who were asked to put the Latin characteristics in order. For this presentation, motion data converted from bvh type to xml type was displayed in three-dimensions, using VR software (Omegaspace). The result of the ranking by 3 subjects was from top down, motion A, motion B and motion $\mathrm{C}$. Thus it was confirmed that the dance motion expresses Latin characteristics. 


\section{Conclusion}

The isolation movement of shoulders and hips in Latin dance was extracted quantitatively. In order to verify whether this characteristic resembled Latin movement, a dance animation with different isolation levels was synthesized, using a motion graph editor. As a result of the evaluation, it was confirmed that Latin movement can be expressed appropriately. In future, we plan to analyze synchronism with music and study getting into the rhythm.

\section{References}

[1] Iwai, D., Toro, F., Inokuchi, S.: Analysis of Dance Motion: Japanese and Latins. In: Proc. SI2002. (2002) 299-300

[2] Kamisato, S., Yamada, K., Tamaki, S.: Sensitivity evaluation and three-dimensional motion analysis of arm motion in dance. (2004) 1-4

[3] Saito, S., Imoto, T., Nakajima, M.: Motiongraph: a Technique of Action Generation for an Autonomous Character. (2002) 22-29 